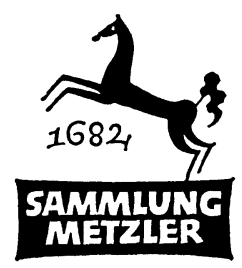

REALIEN ZUR LITERATUR ABT. G: DOKUMENTATIONEN (Reihe b: Zu Unrecht vergessene Texte) 


\title{
ANTHOLOGIE \\ AUF DAS JAHR 1782
}

\author{
HERAUSGEGEBEN \\ VON FRIEDRICH SCHILLER
}

\begin{abstract}
FAKSIMILEDRUCK
DER BEI JOHANN BENEDICT METZLER

IN STUTTGART

ANONYM ERSCHIENENEN ERSTEN AUFLAGE

\author{
MIT EINEM NACHWORT \\ UND ANMERKUNGEN HERAUSGEGEBEN \\ VON KATHARINA MOMMSEN
}
MCMLXXIII
J.B. METZLERSCHE VERLAGSBUCHHANDLUNG
STUTTGART


Die Württembergische Landesbibliothek in Stuttgart stellte dankenswerterweise ihr Originalexemplar für die Reproduktion zur Verfügung.

ISBN 978-3-476-10118-1

ISBN 978-3-476-03836-4 (eBook)

DOI 10.1007/978-3-476-03836-4

M 118

(C) Springer-Verlag GmbH Deutschland 1973

Ursprünglich erschienen bei J.B.Metzlersche Verlagsbuchhandlung und Carl Ernst Poeschel Verlag GmbH in Stuttgart 1973 


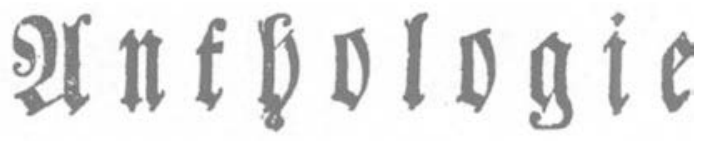

$$
\mathfrak{a} \mathfrak{u} \mathfrak{D} \mathfrak{a} \mathfrak{s} \mathfrak{J a b} \mathfrak{r}
$$

I 782 .

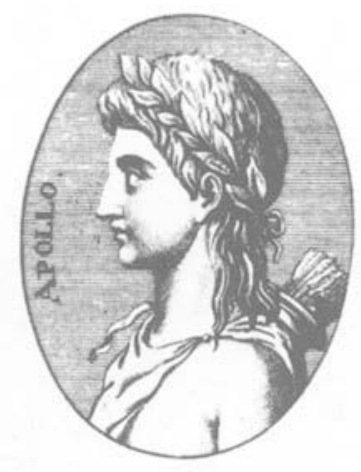

Georuft in oer $\mathfrak{B}$ udoruferei ชั হObOIBlD. 
Meinem \$rinģipal

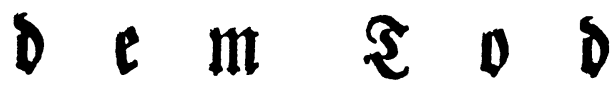

3ngefक̆rieben. 


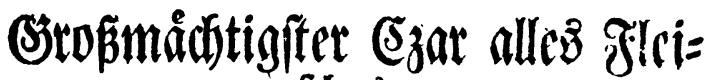 f(t)ez,

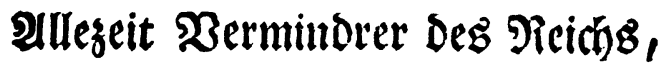 \\ Unergrůndlic)er Nimmerfatt in Der ganzen Natur!}

3) it unterthánigftem Shautfhauern uns terfange ith mich Deiner gefrásigen Majeftít Elappernde Plyalanges zu kúfen,

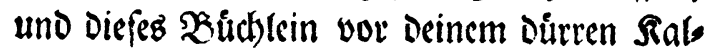
faneus in Demut nieverzulegen. Sjeeine Worginger baben immer bie $\mathfrak{B}$ cife gebabt ibre Gád)lein und gudflein, Dir glcichfam recht vorfeglidid jum 2lerger, bart an beio

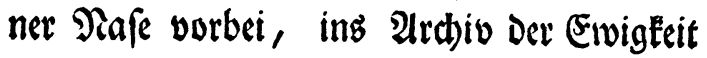
tranzportiren zu laffen, und nidjt geoad)t, Dak fie Dir cben Dnourd) um fo meler Dis Mraul Darnach wdffern madten, Denn aud) an Dir wito Das Sprictbroort netht zum signer: (Beffollen $\mathfrak{B r o d}$ fdomecte gut. Siein! Des Diziren will id Dir's lieber, fo bin id) Dod)

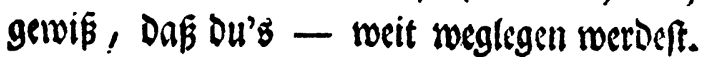
$x_{3}$ Did) 
Tod) Epan bcifcite! - Şd benfe, wir zmeen Eennen uns genauer, Denn nur vom Sjórenfigen. Einverleibt Dem áftulas piicten Droen, Dem Erftgebornen nus Der siuchfe Der Janoora, Der fo alt ift alb Der Gindenfall, bin id) geftanien an Dcinem allture, babe, wie ber Eolyn Şamilfars Den fieben Saígel'n, gefdyoren unferblid)e Feljoe Deiner Erbfeindin Patur, fie zu bee lngetn mit Medifamenten Saceresfraft, eine SBuginburg al fdlingen um dic Grablifbe Eecle, aus Dem Scld zu fhlagen mit Gturm Die Srajige die Deine Gporteln fatmálert, uno Deine Finanzen f(b)wád)t, uno auf Dem

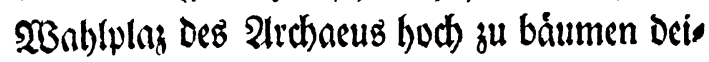
ne mitternád)tlidje Sireujftandarte. - Das fir nun (Denn eine (F)re ift wertl) Der an: Dern) wirft ou mir aubiourfen oen fofftichen S $\mathfrak{a} l \mathfrak{i} \mathfrak{s} \mathfrak{m} \mathfrak{n}$, Der mich mit beiler Saaut uno ganjer 200 olle an Şalgen und Sias De voribergeleitet -

$$
\text { Jusque datum fceleri - }
$$

(1) ja Doch! Shue taz goldiger Maejes naš; ocnn ficlfif Du, id) moddte Dod) nidft 
gern, Dás mirs gienge wie meinen tolleủb: nen Sollegen uno Settern, dic mit Etilet und Eaftuffer bervaffict in finftern Şohb: wegen $\mathfrak{S}$ of balten, voer im unterirrolfben

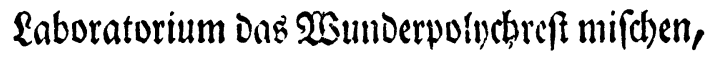
Daz, wenns.búbfd) fleifig genommen wiro, unfere pofitifde Siafen, ibber furz oder lang, mit Sbronvakituren und Etantsfictern Fis zelt. - D'amiens und Siavaillac! - $\mathfrak{H} u$ ! bu! bu! — E⿱ $\mathfrak{b}$ ift cin gut Ding um ges rade GHlieder!

Db́ bu aud Deinen Babn nuf Dftern uno Michaeliz gewezt haft? - Die grofe Buidlerepiocmie in Seipzig uno Franls furt! - Iud) beifa Dürrer! - wiro ein fóniglid) Freffen geben. Deine fertigen Máfler, Zúllerel) und Brunft liefern dir ganje Frndten aus Dem Sabrmartt Des ses benz. - Eelbft Der Eibrgeiz Dein Girofpas Ha, Frieg, Şunger, Feuer und $\mathfrak{X e f t}$ deine gewaltigen Ginger baben dir fdjon fo manche fette Senfibenflopfiago gebalten - Seiz und Gjoloburft Deine mádbtigen Sillermeifter trinken Dir ganze fdiwimmende Stáde im )$(4$ fprus 
forudernoen Sield Dez 2 eltmeers zu. -

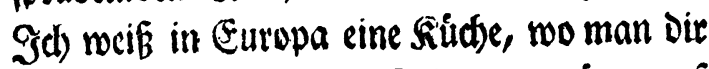
Die rareften Gierictste mit Jefttagsggeprånge auf Die Sufel gefezt bat - Und Dod) - wer bat dide ie fatt gefeben, ooer iber Snoiges ftionen Elagen gehgett? - Eifern ift Deine Serdaunng; grundlos Deine Bsedarme!

Jull - Jd) batte dir nod) fo mand es zu fagen, aber id tummle mich), Daf id) wagfommc - Du bift cin garftiger Sduma: ger - $(\mathfrak{s i b}-\mathfrak{D u}$ mudeft bir Siect)nung, hisre idb), eine (Sieneralcollizion zu exteben, wo dir (Sjrof uno Silein, Meltfugeln uno

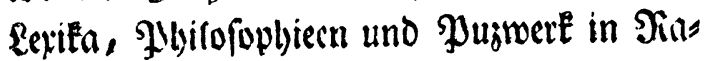
Wen fiegen follen - Sutten 2lppetit, wenns fo meit fommt! - Dod), Shungerwolf Der ou biff! ftele zu, oari bu bich oa nicht

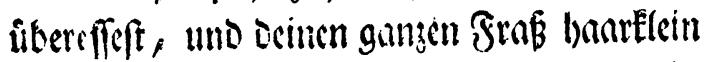
roiedergeben múfeft, wie dir's ein gewiffer 2tthenienfer, Der dir gar nidt wobl will, proplexcint bat.

\section{Y.}


Tobolak ben 2, Februar,

- Tum primum radiis gelidi incaluere Triones. -

SRlumen in Gibirien? - Dabinter fteft sine Sdelmerel), ober bie Sonne muß́ Front gegen Mitternacbt maden. - „U Uno ood wenn ibe eud auf ben Siopf frelltet! $\mathcal{E}_{\mathfrak{g}}$ if nid)t anders; - Wir baben lange genug 300 bel gefangen, laßst's uns einmal aud) mit Blus men verfuden. Sino nidit fajon Enropáer genug gu uns Stieffôhnen der Sonne gepoms men, uno burd) unfern humbertiąbrigen $\bigodot_{\text {dque }}$ gematet, Irgeno ein befaceidenes Shlimden ju fflúcfen? Sdjande unfern 2 dhnen - wir wol: len fie felfft fammeln, und einen ganzen אorb yoll 
voll nad) Europa franliren. - Bertretet fie nidft, ihr Sobne bes milberen Şimmels !

भber im Ėmft fu reben - Das eiferne (Samidht bes wibrigen Borurtheils, bas (a)wer über sem Norben bruitet, von ber Stelle zu råumen, foberte einen ftårferen $\mathfrak{S}_{\text {gebel }}$ als ben Enthufiafmus einiger wenigen, unb aud) ein fefteres Şzpomodition, als bie Saultern yon zween ober bren Patrioten. Dod wenn ffion aud biefe $\mathfrak{A n t h o l o g i e ~ e u c h ~ T e l e r b a f t e ~ E u r o p a ̊ e r , ~}$ fo wenig, als - wenn id den fall feze unfer Mulenalmanad), ben wir - wenn id) ja ben Fall Fezen wollte - håatten losnnen ges parieben baben, mit uns Saneemánnern ver: fógnen wird, fo bleibt ifgr bod mindeftens das Rerbienft, Şand in Şand mit ihren" Famerås binnen im weitentlegenen Teut(d)land Dem ausrddelnden (Sefamad ben (O)nilfang geben 
fu helfen, wie wir Tobolsfianer ju fpredsen belieben.

Wenn eure Scomere im $€$ djaf reden, und eure Şerlules Múlen mit ifren Reulen erfðhla: gen - Wsenn jeder, der feinen bezaflten Sdimerz in Reidenalerantriner ausgutropfen verf(teht, , Das für eine $\mathfrak{B}$ ofazion auf Den Şeli: fon auslegt - wirb man uns Nortlánbern verdenfen mitunter aud in ben Renerflang ber Mufen fu Elimpern? - Eure Mlatadore wollen Sillbergelo gemúnjt haben, roenn fie ige Bruftbilo auf elendes Meb̂ing praigs ten; - und zu Foboldto werden bie Falfd) munzer aufgebangen. Şwar modt ibr oft aud) bei uns Papiergeld fatt rufifaden $\Re$ Rus bils finden, aber Sirieg und theure Zeit ents losuldigen alles.

So geh bann bin, Sibitifde Anthologie (Seb) - Du wirft manden Süpling befeeligen, wirft 
wirft von ifgm auf ben Nadttifd peiner Şerzs eingigen gelegt werben, und zum Dank iffe alabafterne Lilienfdhneeband peinem zåtlis

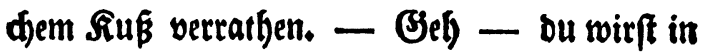
Den 2 (ffembleen uno Stabtvifiten mandben gåf): nenden Sdlunb der Rangenweile ausfúllen, und vielleidgt eine Circaffienne ablofenn, bie fí) im Plazregen ber \&áfterung múbe geftans ben hat. - Geh - bu wirft bie Kưdje mans der Rritifer berathen; fie werden bein \&idjt flieben, und fid gleidh ben Såuglein in beinen

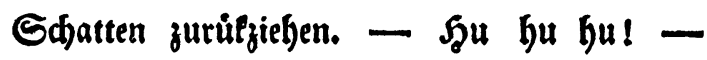
Sdon bơr id Das obrzerfezende Beheule im unwirtbbaren forft, uno buille midh angftvoll in meinen Bobel. 


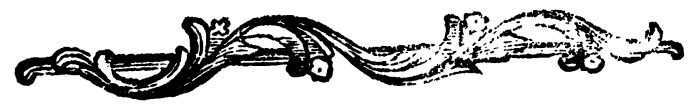

\section{$\mathfrak{s} \mathfrak{n} \mathfrak{n} \mathfrak{a} \mathfrak{l}$ t.}

Sie Journaliften uno Minos 178r. Seite $x$ Santafie an Saura

Bacthub im నriller

2In Die Eonne 12

faura am Slavier 16

Die Sherrlid)teit ber Sdjopfung, eine gantafie 22 Elegie auf Den Tod eines șưnglings 25

Der wirtb[d)aftlidbe בod

32

Roupeau

An ben Salgen zu (d)reiben

33

Die ferligen Mlugenblife an Saura

37

Spinoza

Die Rinosmorberin

2ufid)rift einer frurttengruft

In einer $\mathfrak{B a t a i l l e ~ v o n ~ e i n e m ~ D f f i z i c e ~}$

38

Orrabjarift

4I

42

48

49

59

2) 


\section{IIntbalt.}

III Die Parzen

Seite 54

Der Triumf ber fiebe, eine Symme

58

Slopftod uno IBielano (als ibre Eillyouetten neben simanda biengen)

(jeipriach)

2̧ergleidung

Die Radbe ber Mujen, eine Aneldote som Sycilicu

Dns (Shlik unt bie $\mathfrak{X e i s h e i t}$

Fiazel

76

Qtu einen Moralifiten, Fringment

(srabictrift eines gewiffen - \$hyfiognomen

Eime feidjenfantafie 1780 (in Mujił zu haben bein ŞeranEgeber)

$8 \mathrm{I}$

24eidulus

Ser hypodjonbrifale Pluto, Romanze

88

Die 23 üe

Afftåon:

100

Zైerfid)t ber Unfterblidkeit

100

Borwurf an Saura

IOI

Die Alten und Nenen

105

Der einfáltige Bauer

106

Eogat an $\mathfrak{H}$ (u)de

107

Eitten unb Zciten

109

Ein $\mathfrak{B a t e r}$ an feinen Solgn

110

Die Mep̧iade

III

DB̧ianz Sounengejang * aus bem Sebidite siartbon (in smujit zu haben beym sters aubgeber) 


\section{Innbalt.}

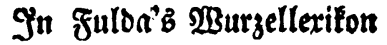

Seite 114

- Siaftraten unb Rånner

115

Doftor Pandolf

122

2n ben frithling

123

Polizeyoromung

124

Die alten unto neuen Şelden

125

Unteridhicb bar Zeiten

125

Sayme an ben unenolid)en

126

2uf Den Setrn $\mathfrak{R} *$

127

Die Birofe ber $\mathfrak{\text { BSelt }}$

128

Segruindete Furdbt

130

Paffantenjettel am Thor ber J̧dllen

I3r

Meiine Blumen

132

Fłud) eines̉ Eiferfůd)tigen

134

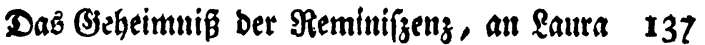

Oruppe aub bem Tartarub

Die Fremtufd)aft, (aub ben Briefen Sultue an Raphael; cinem nod ungebruften Noman)

2In fanuiy

Gefübl am erften Sltober $178 \mathrm{x}$

Peter

162

Der Birtemberget

162

2Ln mein Iáubden

I63

Meland)olie an Saura

166

Die \$eft, eine Fantafie

173

Dab Muttermabl

174

Die 


\section{Innbalt.}

Die Spinne unb ber Seibenwurm Seite 175

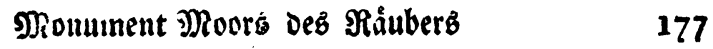

2hư Chloeś Gieburtstag oen 4. Sanuar I8I

Morgenjuntaïe $\quad 184$

lies citteż abwejenden $\mathfrak{B r a ̊ u t i g a m s ~} \quad 187$

An פiinna 190

Der Unterídied $\quad 193$

Slifium, eine Rantnte $\quad 196$

Quirl $\quad 198$

Semele, cine lyrijde Dperette yon zwey Sce: nei 199

Die Buichje ber Manbora 243

Die jdhlimmen Monard)en 244

Graf Eberbard Der Sreiner yon $\mathfrak{B r}$ irtemberg 25 I

Alte Yungfern $\quad 257$

In Gott 258

- Barurenftåndden 260

Der Eatyr' uno meine Mure $\quad 263$

Die Minternad)t $\quad 268$ 